\title{
The Impact of Multimedia Training on Mental Models of Simple Processes
}

\author{
Johnny L. Washington and Erika Rogers
}

\begin{abstract}
The purpose of this paper is to describe work in progress on a project which is designed to study how multimedia training may impact people's mental models of simple processes. A tutorial is under development which helps people learn how to construct an abstract model of the steps needed to produce a letter on a typewriter, and how to change this model when the task moves to a computerized setting. Subjects have been recruited to participate in this study, and the current status of the project is discussed.
\end{abstract}

\section{INTRODUCTION}

Today, large numbers of low level or mechanical jobs are being converted to computer-based technology. In the interests of economic survival, people must quickly learn to function under the new circumstances. Even simple jobs such as typing and filing in an office environment now require very different skills than they did in the recent past. However, many people are overwhelmed by the idea of the new technology, and "fear of computers" has become a common ailment, which increases the challenge of change. The question therefore arises: what causes such fear in this environment, and can we utilize technology itself to overcome this problem?

Our first hypothesis is that, in order to perform traditional mechanical tasks, it was not necessary to develop complex mental models of the task in order to function adequately. In fact, it is possible that one could actually "get by" with a partial or incomplete model by just memorizing the steps needed. First of all, the process itself was simpler - fewer things could go wrong, and there was a limited number of steps involved. Moving the same task to a computer environment, however, requires understanding how the task process fits in with the computing process, and suddenly, there is a need for a more concrete and complex process model. Rote memorization of the steps needed will no longer suffice, since the essence of the computer is to be flexible and extendable in capability. It is our contention that people fear the computer because of this lack of a process model, and the fact that they have not previously had the training or experience to easily generate such a model.

Our second hypothesis is that multimedia technology can help people learn the following concepts:

- how to abstract a mental process model of a traditional task such as typing a letter using a typewriter;

- how to change that process model to represent the same task in a computerized word-processing environment;

- how to relate the new process model to carrying out the task in the real world.

This paper describes an ongoing study to test these hypotheses. The design of the multimedia tutorial used to provide this kind of training is presented, and preliminary experimental results with human subjects are reported.

\section{BACKGROUND}

With new software and more advanced technology being developed on a daily basis, the need for human interaction is becoming even more rigorous and demanding. As a result the need for a strong foundation in the use of computers and software is ever more important. As a means of dealing with this issue, we have embarked on a study that will in essence use the many advances in technology with multimedia software as a medium [6] to help people develop the skills required to properly use a computer as a tool. In keeping with the issue of avoiding "behavioral rigidity" [4], it becomes necessary to provide training that will give an individual the skills needed to complete multiple tasks that are based on a single original task. To maintain this idea, we looked at the results of a study conducted at Carnegie Mellon University. The subjects were asked to review a series of pulley systems 
and decide which system would require the least amount of work. The results showed that the subjects who scored high on the evaluation had clear ideas of the rules and could identify the attributes relevant to the operation of a pully system[1]. In short, subjects who scored high on the evaluation had a stronger foundation in the areas that were relevant to the questions at hand. Just as the latter study showed that a strong foundation in the needed areas was helpful [2], our efforts are focused on providing individuals with the needed skills to traverse smoothly from one task to another. Also, it is expected that by using multimedia as a learning tool, some of the adversities of traditional learning [5] will be eliminated.

\section{APPROACH}

It was decided to explore these ideas by constructing a multimedia tutorial based on the very simple task of typing a letter. This is a basic skill which is needed at the beginning of computer literacy, and most people should be familiar with how to do this task manually (i.e., on a typewriter). The tutorial is designed to help people learn how to formulate a mental model of the more familiar process of using a typewriter, and then to make the transition to the less familiar way of performing the task on the computer, through graphics and animation. Our basic approach is to lead the student from a "real world" or concrete visualization of the task through the use of video, to an abstraction of the task in the form of a flow chart. It is this latter model which is expected to help in the development of the final mental model of the process.

\section{Subjects}

The target population of this study was people who had little or no computer experience, and therefore the Job Training Center of the Atlanta Union Mission was contacted to recruit subjects. They selected a number of candidates who met these requirements, and after providing an initial questionnaire to be distributed, we received feedback from 25 subjects, 14 females and 11 males. The average age of the subjects is 36 years old, and a majority (19) are from minority groups. Half of the subjects have a high school or equivalent education, while nine of them have one or more years of college education; 18 are currently unemployed. When surveyed about computer usage, 16 indicated some exposure to computers, while nine had never used a computer at all.

\section{Methodology}

The overall study has several stages:
1. Survey subjects on their current understanding of the steps needed to a) produce a letter using a typewriter, and b) produce a letter using a computer. This includes asking them to list the materials needed and the steps to be followed, and to draw a picture of the steps if possible.

2. Design and implement a multimedia tutorial which demonstrates the relationships between the real world representations of the two tasks using video, and abstract representations of these tasks in the form of flowcharts. Using animation techniques, the tutorial also demonstrates how to move from understanding the known process (using typewriter) to the unknown process (using computer).

3. Collect data (video and audio) of subjects using the tutorial.

4. Design and implement a suite of tests in which the subjects can demonstrate their understanding of the different parts of the tutorial.

5. Design and implement a suite of tests in which the subjects can demonstrate their ability to actually perform the computer task of producing a letter after interacting with the tutorial.

6. Analyze data and evaluate tutorial components.

7. Redesign tutorial for actual job training applications.

In the remainder of this paper, the first two stages of the methodology are discussed.

\section{DESIGN OF TUTORIAL}

The overall structure of the tutorial includes both interactive and passive elements, thus providing the opportunity for the subjects to learn in several different modes. Although a number of our subjects have had some previous computer experience, it is not clear whether they are familiar with direct manipulation interaction using a mouse. Since the rest of the tutorial depends on this type of interface, an introductory interactive screen is provided which helps the subjects learn two behaviors: a) how to "point and click" to select objects for navigation from one level of information to another, and also to invoke command buttons; and b) how to "drag and drop," which allows objects on the screen to be relocated (this is needed for the post-tutorial tests).

There are four main representation components of the tutorial itself: i) a video of the real world typing task $\left(R_{1}\right)$; ii) an abstracted model of this process in the form of a flowchart $\left(A_{1}\right)$; iii) a video of the real world computing task $\left(R_{2}\right)$; and iv) an abstract model of this process in the same flowchart format $\left(A_{2}\right)$. Each flowchart has two levels of detail describing the process: the first level 

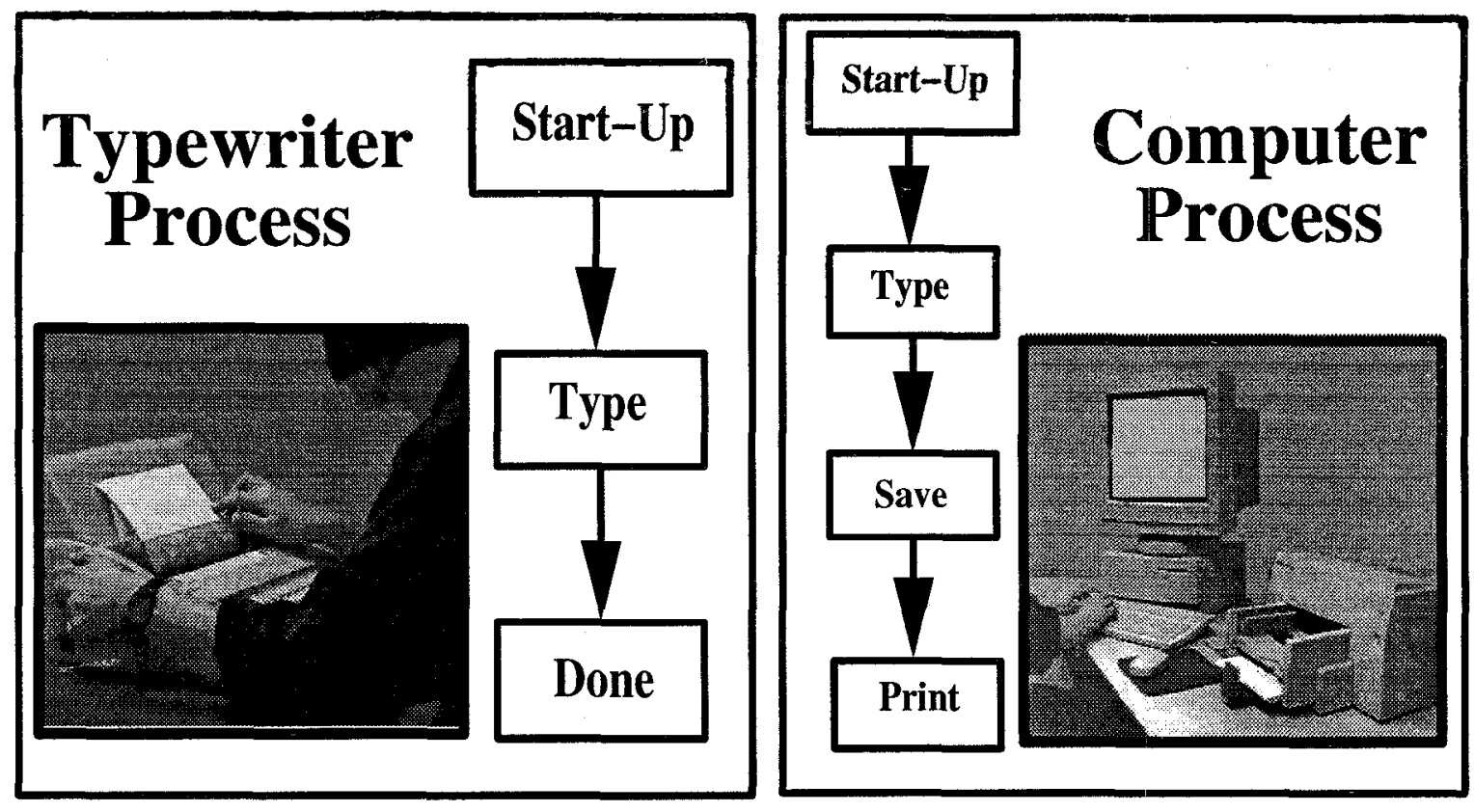

Figure 1: Four Task Representations

just gives the major steps involved in sequential order, while the second level provides a more complicated view of each step, including decision steps as well as loops. An example of the four main components is shown in Fig. 1.

First the subject is shown a narrated video of how a letter is created on a typewriter. After showing the video once through, it is played again, this time with each important step freeze-framed and then mapped to an element of a flowchart. At the end of the process the first level flowchart of the typing process is assembled. The video window becomes a thumbnail, and the flowchart now becomes the main focus of attention on the screen. At this point, a known task has been introduced in a familiar representation, as well as the same task in the unknown abstract representation. Now the subject must make the transition to understanding the unfamiliar task of producing the letter on the computer, and two kinds of presentation order are under consideration.

The first, shown in Fig. 2, is called a vertical presentation style, and is designed to show real to abstract in the known task, followed by real to abstract in the unknown task, followed by a connection between just the abstract flowcharts themselves. The main advantage of this order is the consistency in the presentation of information from the concrete or real (R) to the abstract (A) for both tasks. Also by displaying the connection between abstractions at the end, the repetition might be useful for reinforcement (i.e., they see the abstract representations twice.) A disadvantage of this order is that there are more steps to follow, and also there is a discontinuity between the abstract representations which is only reconciled at the

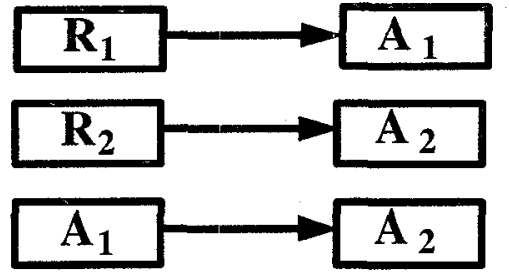

Figure 2: Real to Abstract Representations I

end.

The second presentation style relies on a horizontal ordering of display, as shown in Fig. 3. In this case, the subject is shown, through animation, how the flowchart of the known task changes into the flowchart of the new, unknown task. After this animation, the new flowchart becomes the center of attention, and the individual steps are then animated into the video of someone performing the task on the computer. The major advantage of this approach is that, through continuity from one abstraction to the other, subjects may learn how to manipulate their mental models to understand a new process, rather than always relying on a real world demonstration.

When all four components of the tutorial have been shown to the subject, an interactive learning phase begins, where the two high level flowcharts appear side by side, as shown in Fig. 4, and the subject may freely select any step for which a more detailed view is desired.

At this point, the subject may use the mouse to select any part of the flowchart to see the second level of detail. A more complex flowchart appears, together with 
Figure 3: Real to Abstract Representations II
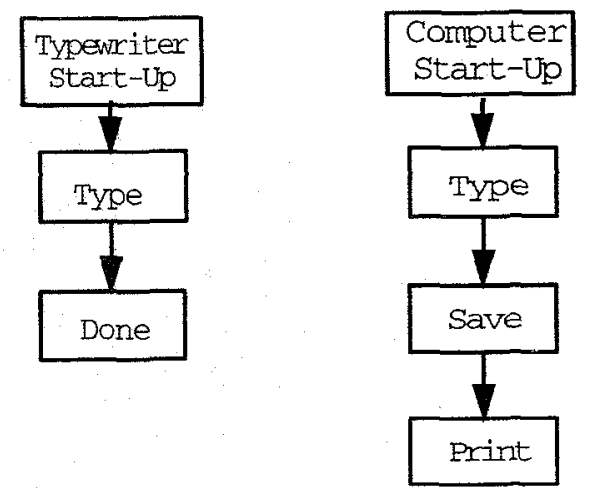

Figure 4: Level 1 Flowcharts

a voice explanation of the step and a photo taken from the video. Fig. 5 shows a comparison of the two secondlevel flowcharts for the "Type" step of each of the process models.

Although there are few major differences in the process of creating a letter on a typewriter as compared to a computer, the overall process contains several steps that should be noted as being available on a computer but not on a conventional typewriter. For this, it is important to note that the use of sound in the multimedia presentation allows the operator to hear things that may not be obvious in the pictures and text. (For example, in the Print step, if there is no printer attached to the computer, a hard copy of the letter cannot be obtained, but the letter information may still be on disk due to the Save step. When using a typewriter, one has a hard copy of the letter just by the mere fact of typing.)

Each subject has unlimited time to complete each tutorial, and is allowed to go between diagrams as many times as necessary to gain a complete understanding. After completing the overview of all processes, the subject is tested by requiring several of the flowcharts previously reviewed to be recreated using a drag and drop method. Fig. 6 shows a sample screen from the tutorial.

\section{PRELIMINARY RESULTS}

When the first set of subject questionnaires was analyzed, we discovered that some of our basic assumptions needed to be reconsidered. In particular, although "computer-phobic" subjects or people with negative feelings about computers were not specifically sought, it was thought that at least the people with no computer experience at all would feel some hesitation or fear about the

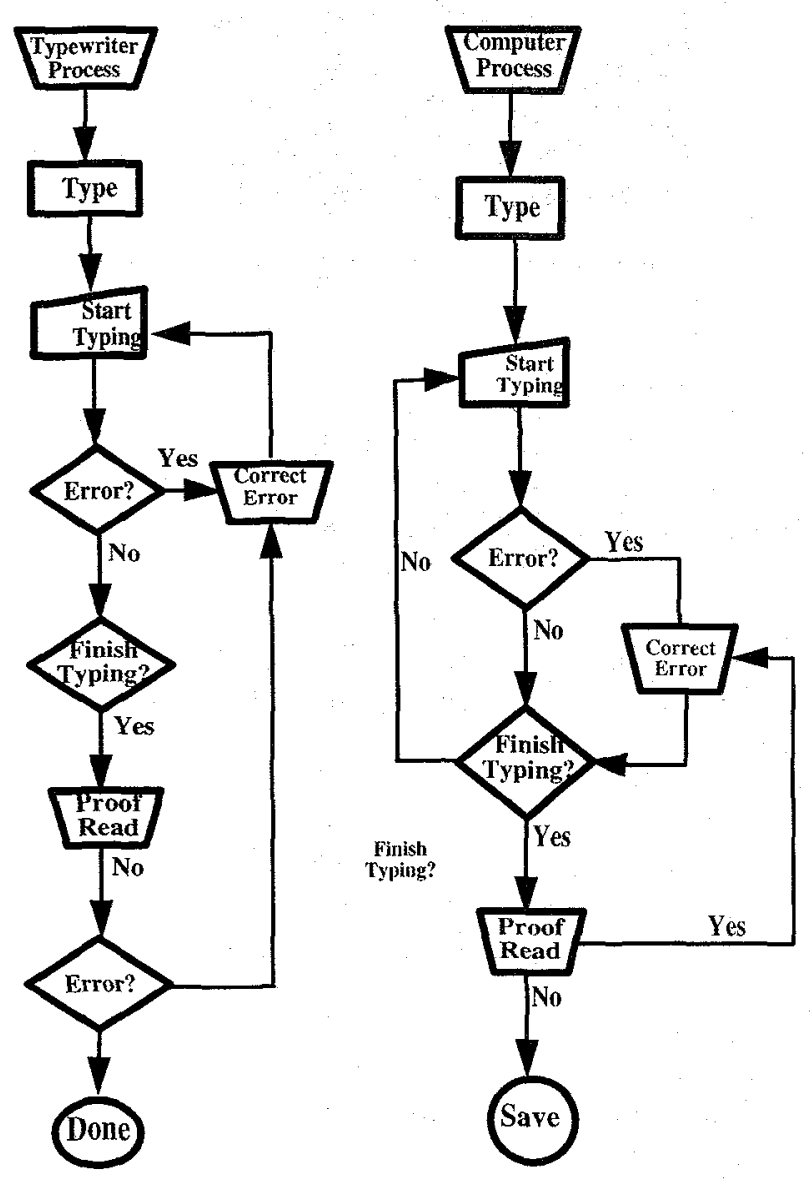

Figure 5: Level 2 Flowcharts for the "Type" Step 


\section{Typewriter Process}

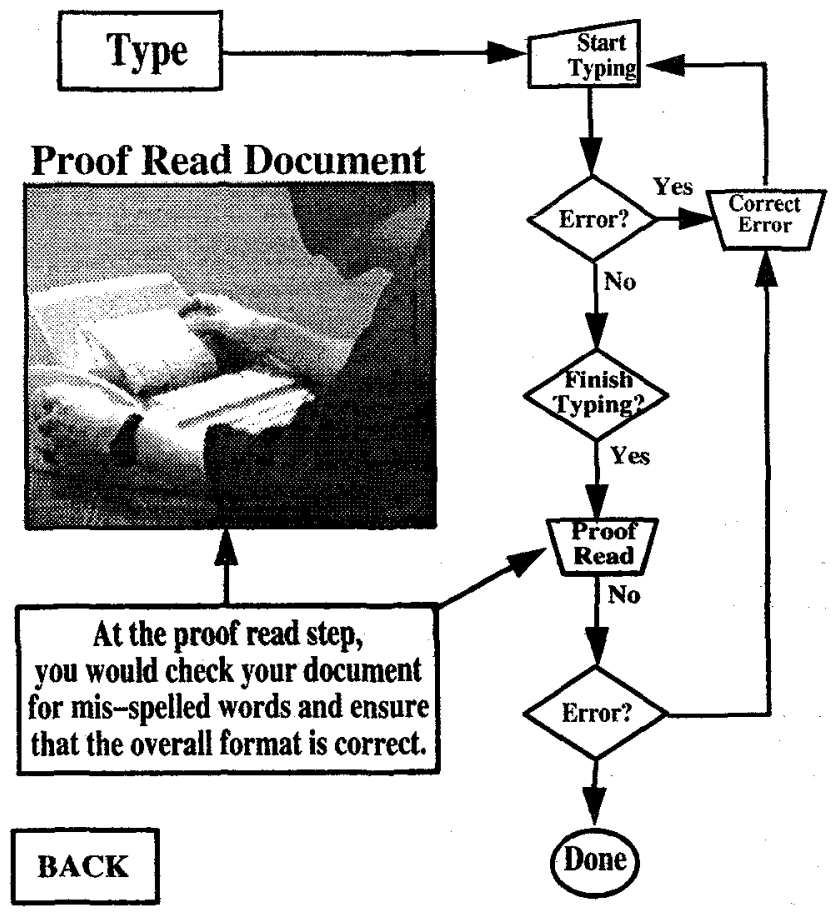

Figure 6: Sample Screen from Multimedia Tutorial

new technology. However, the actual results were somewhat different: a total of 16 subjects indicated that they either liked or loved using the computer. Of these, 14 had previously had some exposure to computers, while two had had none at all. From the remaining subjects who had had no computer experience, three expressed that they would "like to learn", three indicated they didn't know how they felt about them, and only one subject expressed actual dislike. Unfortunately, this last subject did not really complete the questionnaire, so it's not clear whether he was reluctant to be part of the study for other reasons. Of the two subjects who had had previous experience but said they had "other" feelings about the computer, both used words like "complicated" and "frustrating" when describing reasons for their feelings.

The second part of the questionnaire was designed to elicit informally how the subjects think about the two tasks: first they were asked to list the objects needed to create a letter using a typewriter, then to list the steps they would take to perform this task, and to draw a picture of the steps they listed. The first item focused on the "what" part of the task, while the second emphasized the "how". The third was included to see whether any subject would spontaneously generate a diagrammatic representation of the steps. The results from this survey proved to be very interesting.

While most of the subjects had some ideas about the materials needed for the typing task, the reverse was true for the computing task, even though a number of the subjects had previous computer experience. For "things needed" to type a letter, it was expected that subjects would list physical materials such as paper, typewriter, correction fluid and dictionary; what was unexpected was the inclusion of process-related concepts (such as margin setting, heading, greetings, rough draft), and knowledgerelated concepts (typing skills, knowledge of formatting a letter and knowledge about the typewriter). This suggests that even for performing a simple task like typing on a typewriter, some subjects do not have a clear picture of what is needed.

Fifteen of the subjects were not able to list any steps involved in the typing task, while 17 did not list steps for the computer task. This does not necessarily mean that they have no idea of how to perform the tasks, but rather that they have difficulty articulating the step-wise process: (e.g., one response said "just think about how I was going to format it and do it" for the typing task). The diagramming question also produced interesting results: 11 subjects drew a picture for the typing task, while 12 produced a picture of some kind for the computer task. However, in every case, the picture produced had to do with the objects needed, rather than the steps taken subjects drew typwriters and computers, layouts of the desk top, and sometimes a person sitting at the desk per- 
forming the task; but no one drew any kind of abstract diagram of the process.

These preliminary results suggest that while the subjects may have some familiarity with one or both tasks, there is no indication that many of them have a clear idea of what they are doing (at least not clear enough to express it). The subjects' representations of the computer tasks were very similar to those of the typewriter, which also suggests that they do not have a strong model of how these tasks differ. Based on these results, it is felt that the introduction of a more formal "process model" through the multimedia tutorial should help the subjects learn these concepts enough to have confidence in the more complex task.

\section{CONCLUSIONS}

With the introduction of computer technology at almost every level of employment, it is critical that people who are retraining for these types of jobs be given the opportunity to gain a better understanding of what they are doing. This will enable them to not only compete for jobs, but to be more effective once they get the job. Otherwise we face the possibility of more and more high-tech tools with less and less productivity. This paper describes the initial stages of a study which attempts to address these problems. The use of multimedia techniques to help people develop mental models of such simple tasks as typing a letter on a computer may be a promising approach to providing low-cost and efffective training to a large community of job trainees.

\section{ACKNOWLEDGEMENTS}

Support for this work under NSF Grant IRI-9502289 and Technology Reinvestment Project TRP \#1304, NSFDUE-9453883 is gratefully acknowledged. The authors would also like to thank Ms. Angela Scoggins from the Atlanta Union Mission for her kind assistance.

\section{REFERENCES}

[1] K. Forbus and D. Gentner, "Learning Physical Domains: Toward a Theoretical Framework." In R.S. Michalaski, J.G. Carbonell, \& T. M. Mitchell (Eds), Machine learning: An artificial intelligence approach. Los Altos, CA: Morgan Kaufman, 1986, pp. 311-34.

[2] J. Hedberg, B. Harper, C. Brown, and R. Corderoy, "Exploring user interfaces to improve learning outcomes." In I. Beattie, C. McNaught and S. Wills (Eds.), Interactive multimedia in university education: Designing for change in teaching and learning. Amsterdam: Elsevier, 1994, pp.15-29.
[3] M. Hegarty, M. Just and I. Morrison, "Mental Models of Mechanical Systems: Individual Differences in Qualitative and Quantitative Reasoning." Cognitive Psychology 20, 1988, pp. 191-236.

[4] J. Holingsworth, B.W. Wei and L. Latour, "MicroAchitecture of Software Components and the need for good Mental Models of Software Subsystems Leaders." http://www.umcs.maine.edu/ ftp/wisr/wisr7/mmwg/ hreport.html.

[5] C. Quinn, "Engaging Learning." http://itech1.coe.uga.edu/itforum/paper18/paper18.html, The University of New South Wales.

[6] S. Tripp, "Media Learning." http://itech1.coe.uga.edu/itforum/paper16/paper16.html, University of Aizu. 